\title{
WACO for Mammogram Classification
}

\author{
S. Vani Kumari \\ Asst.Prof in CSE Dept, \\ GMRIT, Rajam
}

\author{
Sowrya R. \\ GMRIT,Rajam
}

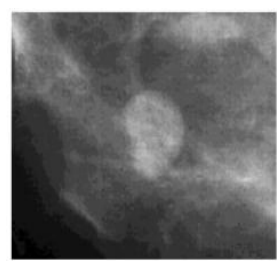

Fig1.(a)

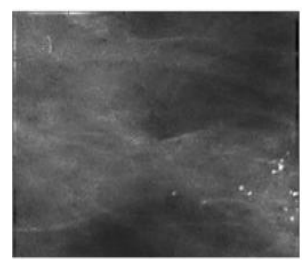

Fig1. (b) (a) Mammogram showing a big mass and

(b) a clustered micro calcification

A mass is a localized assortment of tissue noticeable in two exclusive projections, and calcifications are small calcium deposits. Surprisingly smaller and clustered calcifications are related to malignancy whilst there are different calcifications (diffuse, regional, segmental and linear) which might be more commonly benign. Such calcifications are termed as micro calcifications (MC). Within the early levels of breast melanoma, these signs are defected and thus make analysis by visual ocular observation difficult. Although mammogram bug useful evidence for the early detection of breast cancer, it is hard for radiologists to make precise and steady judgments due to the huge amount of data and extensive screening. Consequently, some cases are missed during the routine check few method are widely used. Some are(NN) (BPN)and SVM. (NN) has two phases one is to identify the suitable structure for the network, and numerical weights for the neuron connections must be efficiently updated.(NN)training procedure as a continuous optimization problem. In the case of weight optimization for neural network training, the optimization algorithms and gradient descent algorithms will choose all the weight connections available in the NN architecture, the constant weight updation and leads to better performance. Here we have an effective architecture and learning problems with a three-layer Backpropagation. Neural Network (BPN) classifier. For a standard BPN training phase, at the end of every iteration, the weights are calculated and uniformly back-propagated and connected to all neurons are strongly affecting the output and those connections could be weight updated. NN uses the six feature to train the data for (MLP) those are contrast, variance, mean, standard deviation, kurtosis, and smoothness. From the loaded image, we calculate the feature for target value and stored in the file. The process is repeated for all images are malignant or benign. The weaver ant's ability to build large nests from living leaves has clear contributed to their environmental success. As this novel ACO revivifying the behavior of weaver ants' nest building actions to bind the neurons randomly between the layers. It is named as Weaver Ant Colony Optimization (WACO). Weaver ants' forms closed nests and the behavior made us to change the architecture of BPN closing the nest, and added an extra connection between the input-output layer of neurons. The pheromones (weights) of the connections between the neurons are randomly connected and network starts the learning phase. Randomly weaver ants are allowed to construct the path (nest) between 
the input-hidden-output-input layers. Texture analysis plays an important role to diagnose normal and abnormal types. Feature extraction can be done by local binary patterns (LBP) [19]. LBP can also useful to discover the face reorganization, shape localization, and dynamic texture recognition It is computed by comparing it with its neighbor's parameter. The objective of preprocessing is detaching an irrelevant noise and improve the quality of an image, and erase unwanted parts in the mammogram. Morphological operators are applied on the binary image before they convert gray image into a binary image. Erosion, Dilation, Top-Hat, and Bottom-Hat are four morphological operations are used to improve the image for further processing. Multilayer Perceptron (MLP)[20] and Support Vector Machine (SVM) for the detection and classification. It classifies are used to compare the classification results. A False Positive (FP) rate is considered a weary in localizing mass in mammogram images. the rejection model based on the Support Vector Machine (SVM) [20] has been using minimized FP rate of segmented images. The rejection model based on the Support Vector Machine (SVM) has been used in decrease the FP rate of segmented images. In order to change the contrast or brightness of an image, improved Contrast tool performs contrast stretching. This process has, pixel values below a specified value are displayed as black, pixel values above a defined value are exposed to view as white, and pixel values in between these two values are showed as shades of gray. The gray-level cooccurrence matrix (GLCM) is a statistical method of probe texture that considers the structural relationship of pixels.

\section{RELATED WORK}

Most of the methods use neural network as the classifier (Varela C. et al. 2007 ; Li H. et. al. 2008 and Fauci F. et al 2004)[1, 2, 6]. Jiang, J. et al. (2007) [7] used the genetic algorithm to systemize the mammograms. Stylger et al. (2011) [8], Jinchang Ren (2012) and Juan F. et al. (2012) [9, 10] used support vector mission for mammogram classifying. The first ACO based algorithm for classifying rule discovery, called, Ant-Miner (Parpinelli et al. 2002) [11]. The information acquired (Entropy) has been used as the heuristic formula value of a term. After the subsequent part of a rule has been constructed, the resultant of the rule is assigned by a majority vote of the training specimen covered by the rule. The constructed rule is then cropped to remove the unrelated terms and to improve its accuracy. The extensions of the Ant-Miner algorithm come up with Liu et al. in Ant Miner2 (Liu et al. 2002)[12] and Ant Miner3 (Liu et al. 2004)[13]. Ant Miner2 uses density roughly calculated as a heuristic function instead of information obtain is used in Ant-Miner. They explain that this heuristic value does the same job as well as the complex one and hence Ant Miner2 is computationally not so much expensive than the original Ant-Miner. Ant Miner3 uses a different pheromone upgrade method with the pheromone of only those terms that occur in the rule and do not evaporate the pheromones of new terms. David Martens et al. (2007) [14] bring forward a Max-Min ant system based algorithm (AntMiner+) that vary from the previously proposed Ant Miners in a number of aspects. Only the best ant is allowed to upgrade the pheromone, the range of the pheromone trail is restricted to an interval, class label of a rule is pick out the prior to the construction of the rule and a different rule feature measure is used. Other works on Ant-Miner involve (Smaldon J. et al. 2006)[15] in which an algorithm is designed for unordered rule sets has been presented. PSO (Holden N. et al. 2007)[16] algorithm is used for unending valued attributes and ACO for formally valued features and these two algorithms are jointly used to build rules. The issue of continuous attributes has also been dealt in (S. Swaminathan 2006 and Otero F. et al. 2008) [17, 18]. The proposed algorithm keep the basic structure of the previous Ant-Miner algorithms. In this proposed method, the interested function uses Tsallis entropy instead of Shannon Entropy measure. A comparative study is made in the field of Mammogram image processing.

\section{WACO}

The block diagram of developed system is shown in the fig.1, which works in two phases, the first phase is Training phase and the second phase is Testing phase for both or two classifiers- BPNN and Weaver Ant Colony Optimization with BPNN, in first phase it takes the MRI images of breast cancer affected patients and perform the image processing techniques shown in the fig. 1 and feature are stored in the knowledge base and it is used for training the neural network, and in second phase evaluate MRI images are taken as an input and perform the same operation we performed before on training images and features of examining images are compared with stored image in knowledge base. The third classifier is Weaver Ant Colony Optimization which takes features of both training and testing images to classify the breast cancer.

\subsection{Image acquisition}

Quite often, scanning an image means making it computationally manageable. When reworking an image to a digital form, it is indispensable to convert it right into a signal. The definition of this signal, which represents the image, is a system the place each pixel is represented by an integer value proportional to the brightness and color of the corresponding point within the image.

\subsection{Image Preprocessing}

Processing tactics are used to support some features of the image similar to mitigating noise and embellishing contours; portion detection; image registration; and enhancing the characteristics of depth, color, and texture.

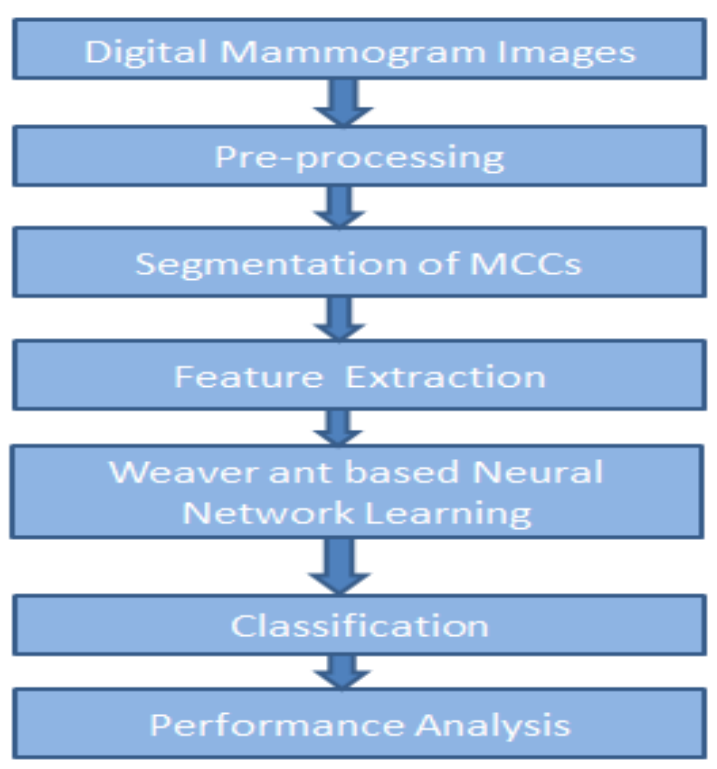

Fig 2.Block diagram of the system

\subsection{Feature Extraction}

The work include extraction of the important features for image recognition. The features extracted give the property of the texture, and are keep in knowledge base. [1]. the extracted features are compare with the unknown sample means the 
testing image for classification. We used a gray level Cooccurrence matrix for texture feature extraction.

Grey degree co-occurrence matrix (GLCM) was once firstly introduced by means of Haralick. A gray-degree coprevalence matrix (GLCM) is practically a two dimensional histogram. The GLCM procedure considers the spatial relationship between pixels of exclusive gray levels. The procedure calculates a GLCM by means of calculating how probably a pixel with a specific depth $i$ happens in relation with one more pixel $\mathrm{j}$ at a exact distance $\mathrm{d}$ and orientation $\Theta$. A co-incidence matrix is distinctive by way of the relative frequencies $P(i, j, d, \Theta)$. A co-occurrence matrix is therefore operate of distance $d$, attitude $\Theta$ and gray scales $i$ and $j$.

We extracted the following texture feature which are given below

Contrast: Contrast returns a measure of the intensity contrast between a pixel and its neighbor over the entire image

$$
\begin{aligned}
& \text { Contrast }=\sum_{i=1}^{k} \sum_{j=1}^{k}(i-j)^{2} p_{i j} \\
& \text { Range }=\left[0(\operatorname{size}(\mathrm{GLCM}, 1)-1)^{\wedge} 2\right] \\
& \text { Contrast is } 0 \text { for constant image }
\end{aligned}
$$

Correlation: Returns a measure of how correlated a pixel is to its neighbor over the whole image. [6][9]. Range $=\left[\begin{array}{ll}-1 & 1\end{array}\right]$ Correlation is 1 or -1 for a perfectly positively or negatively correlated image. Correlation is $\mathrm{NaN}$ for a constant image.

$$
\begin{gathered}
\text { Correlation }=\sum_{i=1}^{k} \sum_{j=1}^{k} \frac{\left(i-m_{r}\right)}{\sigma_{r}} \frac{\left(j-m_{r}\right)}{\sigma_{\mathrm{C}}} p_{i j} \\
\sigma_{r} \neq 0, \sigma_{c} \neq 0
\end{gathered}
$$

Where $\mathrm{mr}$ and $\mathrm{mc}$ are mean computed along rows and columns respectively, are in form of standard deviations computed along rows and column respectively.

Angular Second Moment(Uniformity or Energy):- Angular Second Moment is also called as Uniformity or Energy. ASM is sometimes used as texture measure. It is the sum of squares of entries in the GLCM ASM measures the image homogeneity. Angular Second Moment is high when image has very good similarity or when pixels are very similar.

$$
\text { Energy }=\sum_{i=1}^{k} \sum_{j=1}^{k} p_{i j}{ }^{2}
$$

Rang $=[0,1]$ Energy is 1 for constant image

\section{Weaver Ant Colony Optimization based Back propagation} Neural Network:

The algorithm for the proposed Weaver Ant Colony Optimization based Back propagation Neural Network (WACO-BPN) learning is given in Algorithm 2.

Inputs: Data Samples, $\mathrm{D}_{\mathrm{ij}}$ with the Class Labels $\mathrm{C}_{\mathrm{i}}$

Output: Weight Optimized Neural Network Architecture.

a) Choose the number of neurons in input, hidden and output layers.

b) Construct a closed BPN with 3-layer Architecture.

c) Choose the samples for training, $\mathrm{D}_{\mathrm{T}}$ d) Normalize the feature vectors of each attributes between $[0,1]$.

e) Connect the neurons from one layer to another with connection weights.

f) Initialize the pheromones (connection weights) randomly between $[-0.5,0.5]$

g) Start the Training

h) For each Sample

i. Calculate the output of the BPN with current weights and store the error (Gmin)

ii. Choose the number of weaver ants $\mathrm{N}_{\text {wa }}$, where $\mathrm{N}$ $\mathrm{w}_{\mathrm{a}} \leq \mathrm{N}_{\mathrm{a}} \leq \mathrm{N}_{\mathrm{C}}$

iii. Place the ants randomly to bind the neurons at input-hidden-output layers.

iv. Calculate the output of the BPN by considering the weights from the neurons which are bounded.

v. Store the error (Lmin)

vi. If $\mathrm{G}_{\min }>\mathrm{L}_{\min }$,

Update the pheromones only for the bounded neurons and evaporate the pheromones for the rest of the connections. And assign, $\mathrm{G}_{\min }=\mathrm{L}_{\min }$

vii. Else Update the pheromones for the unbounded neurons and evaporate the others.

viii. Calculate the error of NN, If error $<0.0001$ then go to step (ii)

ix. Else, Take the next sample and goto step (f)

Classifier Implementation Classifiers are used to group the breast cancer. We implemented three classifiers two categorize the breast cancer which are given below -

BP neural network: Synthetic neural networks are largely used solving problems in inspecting biomedical signals, due to the fact that of their variety of applicability and their capacity to gain data of intricate and nonlinear members of the family. ANNs are trained via illustration data set as an alternative of ideas. When used in diagnosis of neuromuscular disorders, ANNs will not be affected by explanations matching to human fatigue, emotional states, and habituation. They are also equipped of quick identification, evaluation of conditions, and prognosis in actual time. There are various forms and architectures of neural networks various fundamentally in the way they study; the main points of that are good documented in literature. BP neural network is a common multilayer feed forward community trained in step with back propagation algorithm. BP neural network uses parallel transmit processing procedure to manage both qualitative and quantitative aptitudes. It has robust robustness, fault tolerance and adaptability and might entirely approximate any complex nonlinear relationship. 


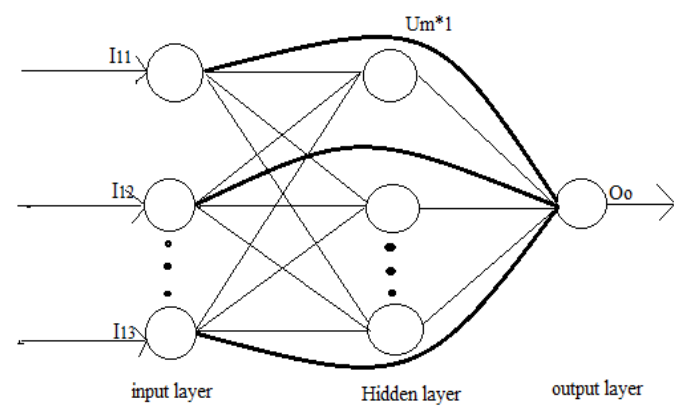

Fig.3. Proposed BPN architecture

The proposed architecture is capable to minimize the magnitude of epochs competitively, although it probably an easy amendment. Because of a different connection is introduced, an extra matrix is presented [U] for maintaining its weights. Identical to the ordinary BPN, [U] can also be initialized with random weights to start with. This modification leads to a transformation in Equation for calculating the input of the output layer $\mathrm{I}_{\mathrm{O}}$ as

$$
I_{O=W}{ }^{T} o_{H+U^{T}} O_{I}
$$

The developed system classifies the mammogram images of breast cancer affected patients. We implemented three classifiers; each classifier has their own effectiveness to classify the breast cancer. Here takes the 3 types of breast cancer mammogram images which are categorize into five different type of class from class I to class III.

Pheromone (beeswax) "chemical substance that animal that spreads in air" value are randomly initialized between [- 0.5 to $0.5]$. Performance is calculated by true or false positive \& negative values.

Analysis: The performance of any classification algorithm can be analyzed using the following four metrics True Positive (TP), True Negative (TN), False Positive (FP) and False Negative (FN). Where TP and TN as the numbers of correctly classified positive and negative samples, FP and FN are

the numbers of incorrectly classified positive and negative samples.

$$
\begin{aligned}
& \text { True Positive Rate }(\mathrm{TPR})=\mathrm{TP} /(\mathrm{TP}+\mathrm{FN}) \\
& \text { False Positive Rate }(\mathrm{FPR})=\mathrm{FP} /(\mathrm{FP}+\mathrm{TN})
\end{aligned}
$$

The proposed method performance is analyzes by Receiver Operation Character (ROC) [20] curves. It analysis and its variants are commonly used for quantitative evaluations of classifiers, especially for the detection and classification of MCCs. ROC curve is a graph between the True Positive Rate (TPR) and False Positive Rate(FPR). When the accuracy increased, the ROC curve covers most of the area in the graph, represents a higher sensitivity corresponds to a lower false positive rate. In this paper 15 images are used for testing out of which 13 images where (TP) and 2 images are (FP). Performance is calculating with $86 \%$ result.
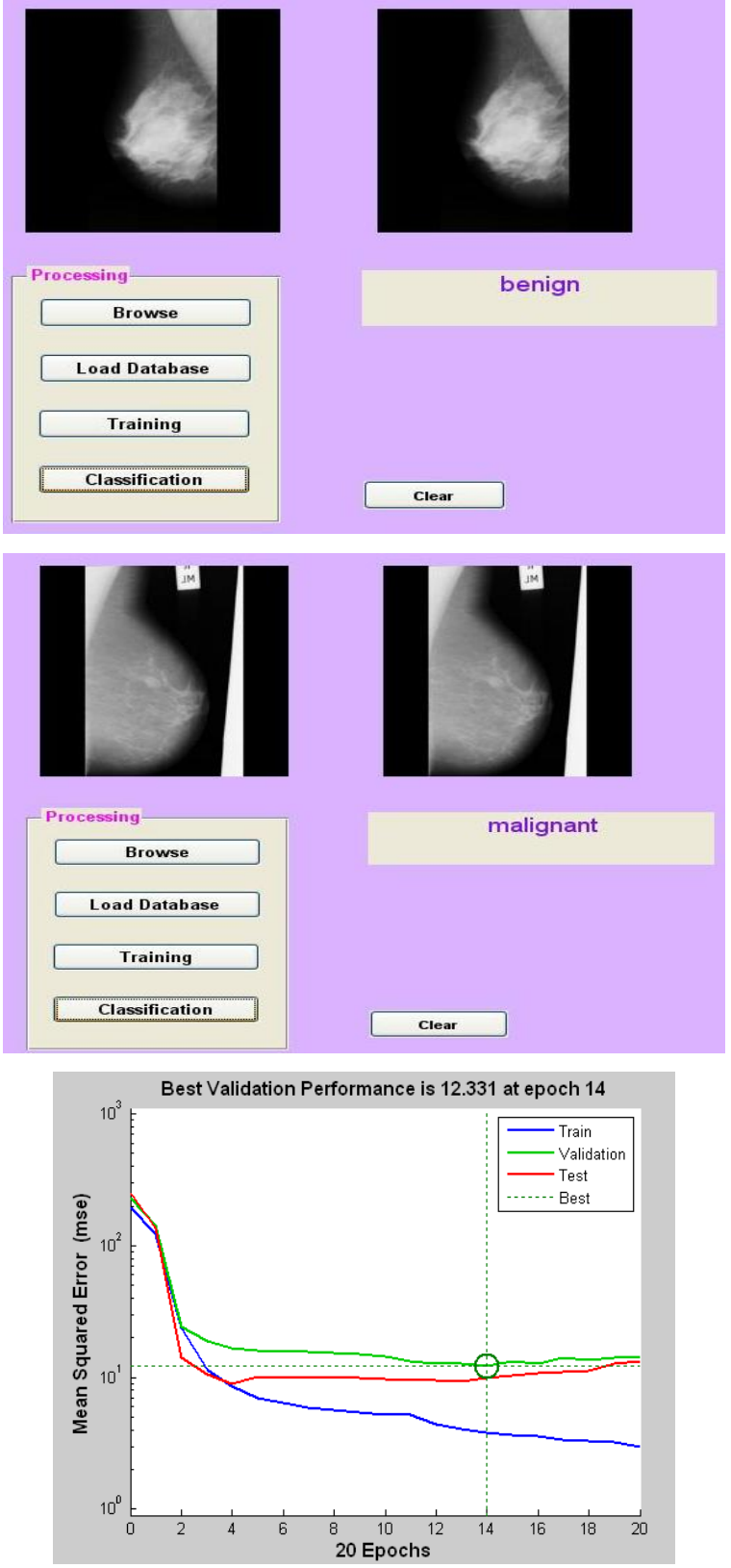

\section{CONCLUSION}

In this paper, improved watershed transformation and binary threshold methods are involved for segmented images. The proposed method uses a Weaver Ant Colony Optimization (WACO) algorithm for weight updation to improve the learning efficiency of BPN which also improves the classification accuracy. In BPN architecture neurons are connected to each other form of input, hidden \&output layers. Waco algorithm simulates the behavior of a special' Weaver Ants' release pheromone (beeswax) chemical substance animal that release in air" ants construct their nests building behavior using the remarkable pheromone and weights are updated for highest weights from the bounded neuron. Update the pheromones for the unbounded neurons and evaporate the other neurons. The breast cancer classification system has been designed by using Back propagation neural network and weaver Ant colony which uses image processing techniques like image preprocessing, image segmentation and for extracting feature Gray Level Co-occurrence matrix to get the haralick feature from mammogram image. The system uses three classifiers for categorizing of breast cancer which are 
Back Propagation Neural Network of WACO and $86 \%$ of accuracy is obtained by using the proposed method.

\section{REFERENCES}

[1] C. Varela, P. G. Tahoces, A.J. Méndez, M. Souto, \& J.J. Vidal, "Computerized Detection of Breast Masses in Digitized Mammograms. Computers in Biology and Medicine,” 37, pp. 214-226, 2007.

[2] F. Fauci, S. Bagnasco, R. Bellotti, D. Cascio, S.C. Cheran, F. De Carlo, G. De Nunzio, M.E. Fantacci, G. Forni, Lauria, A., Torres, E.L., Magro, R., Masala, G.L., Oliva, P., Quarta, M., Raso, G., Retico, A. \& Tangaro, S.(2004). "Mammogram Segmentation by Contour Searching and Massive Lesion Classification with Neural Network," Proc. IEEE Nuclear Science Symposium Conference Record, Rome, Italy, IEEE Press, 5, pp. 2695-2699, DOI: 10.1109/NSSMIC.2004.1462823, 2004.

[3] Jiawei Han \& Micheline Kamber, "Data Mining Concepts and Techniques," Morgan Kaufmann, San Francisco, USA, 2006.

[4] E. Bonabeau, M. Dorigo, \& G. Theraulaz, "Swarm Intelligence: From Natural to Artificial Systems," New York, Oxford University Press, 1999.

[5] M. Dorigo, A. Colorni, \& V. Maniezzo, "The Ant System: optimization by a colony of cooperating agents," IEEE Transactions on Systems, Man, and CyberneticsPart B, 26, pp. 29-41, 1996.

[6] H. Li, M.L. Giger, Y. Yuan, L. Lan, \& C.A. Sennett, "Performance of CADx on a Large Clinical Database of FFDM Images," Proc. IWDM, LNCS, pp. 510-514, DOI : 10.1007/978-3-540-70538-3-71, 2008.

[7] J. Jiang, B.Yao, \& A.M. Wason, "A genetic algorithm design for microcalcification detection and classification in digital mammograms," Computerized Medical Imaging and Graphics, 31, pp.49-61, 2007.

[8] D. Stylianos, E. Tzikopoulos, Michael, V. Mavroforakis, Harris, Georgiou, Nikos Dimitropoulos \& Sergios Theodoridis, "A fully automated scheme for mammographic segmentation and classification based on breast density and asymmetry," Computer Methods and Programs in Biomedicine, 102 , pp. 47-63, 2011.

[9] Jinchang Ren., "ANN vs. SVM: Which one performs better in classification of MCCs in mammogram imaging," Knowledge-Based Systems, 26, pp. 144-153, 2012.

[10] F. Juan, Ramirez, Villegas \& F. David, Ramirez, Moreno, "Wavelet packet energy, Tsallis entropy and statistical parameterization for support vector-based and neural-based classification of mammographic regions," Neuro computing, 77, pp. 82-100, 2012.
[11] R.S. Parepinelli, H.S. Lopes, \& A. Freitas, "An Ant Colony Algorithm for Classification Rule Discovery". Proc. H. A. a. R. S. a. C. Newton, Data Mining: Heuristic Approach, Idea Group Publishing, pp. 31- 35, 2002.

[12] Bo, Liu, A. Hussien, Abbass \& Bob Mckay, "DensityBased Heuristic for Rule Discovery with Ant-Miner, in the joint Workshop of Australia-Japan on intelligent and evolutionary system," pp. $180-184,2002$.

[13] Bo, Liu, A. Hussein, Abbass \& Bob McKay, "Classification Rule Discovery with Ant Colony Optimization," IEEE Computational Intelligence Bulletin, 3, pp. 31-35, 2004.

[14] David, Martens, Manu, De, Backer, Raf, Haesen, Jan, Vanthienen, Monique, Snoeck \& Bart, Baesens, "Classification with Ant Colony Optimization. IEEE Transactions on Evolutionary Computation," 11, pp 651665, 2007.

[15] J. Smaldon, \& A.A. Freitas, "A New Version of the AntMiner Algorithm Discovering Unordered Rule Sets," Proc. Genetic and Evolutionary Computation Conference, Seattle, Washington, pp. 43 -50, DOI. 10.1145/1143997.1144004, 2006

[16] N. Holden, and. A.A. Freitas, "A hybrid PSO/ACO algorithm for classification," Proc. GECCO Workshop on Particle Swarms: The Second Decade, ACM Press, New York ,pp. 1- 11, 2007.

[17] Swaminathan, "Rule Induction using Ant colony Optimization for Mixed Variable Attributes," MSc Thesis, Texas Tech. Univ, 2006.

[18] E. Fernando, B. Otero, A. Alex, Freitas \& G. Colin, Johnson, "c Ant-Miner: An Ant Colony Classification Algorithm to Cope with Continuous Attributes, Ant Colony Optimization and Swarm Intelligence," Lecture Notes in Computer Science, 5217, pp. 48-59, 2008.

[19] S.Vani Kumari, "Breast Cancer Detection using Local Binary Patterns". International Journal of Computer Applications 123(16):6-9, August 2015. Published by Foundation of Computer Science (FCS), NY, USA. BibTeX

[20] S.Vani Kumari,, Dr.K.Usha Rani "Mammogram Classification Using Multilayer Perceptron and Support Vector Machine",International Journal of Computational Science, Mathematics and Engineering

[21] Weaver Ant Colony Optimization Based Neural Network Learning for Mammogram Classification.

[22] A.Kaja Mohideen, Department of Applied Mathematics and Computational Sciences, PSG College of Technology, Coimbatore, Tamil Nadu, India 